\title{
Fluoroscopy-free recrossing of the interatrial septum during left atrial ablation procedures
}

\author{
Nikola Pavlović • Tobias Reichlin • Michael Kühne • \\ Sven Knecht • Stefan Osswald • Christian Sticherling
}

Received: 23 April 2014 / Accepted: 7 July 2014 / Published online: 15 November 2014

(C) Springer Science+Business Media New York 2014

\begin{abstract}
Aim The purpose of this is to evaluate the safety and feasibility of recrossing the interatrial septum in case of inadvertent loss of or need for repeated left atrial access using a simple electroanatomical landmark without the use of fluoroscopy. Methods Twenty-five consecutive patients undergoing pulmonary vein isolation (PVI) for paroxysmal $(n=12)$ or persistent $(n=13)$ atrial fibrillation ablation were included. All procedures were performed using an electroanatomical mapping system (Carto 3, Biosense Webster, Diamond Bar, USA). After fluoroscopy-guided double transseptal puncture and fast anatomical mapping of the left atrium, a reconstruction of the transseptal access was created by retracting the mapping catheter into the sheath to the level of the inferior vena cava. After completing the left sided ablation, both sheaths and catheters were withdrawn to the inferior vena cava. Recrossing was then attempted by fellows (EF) and experienced operators (EO) using the reconstruction of the transseptal access in a standard right anterior oblique (RAO) and left anterior oblique (LAO) projection without the use of fluoroscopy.

Results Using the described technique, EP fellows and experienced operators could recross the interatrial septum without fluoroscopy in all patients. Median time needed for recrossing was $14 \mathrm{~s}$ (interquartile range (IQR) 7-20). Median recrossing times did not differ significantly between EF and EO (14 (IQR $8-26.5 \mathrm{~s}$ ) versus 12 (IQR $6.5-17.5 \mathrm{~s}), p=0.26$ ). In five ( $20 \%$ ) procedures, recrossing was necessary during the procedure after intermittent mapping of the right atrium or inadvertent catheter dislodgment.
\end{abstract}

N. Pavlović $(\square) \cdot$ T. Reichlin $\cdot$ M. Kühne $\cdot$ S. Knecht

S. Osswald $\cdot$ C. Sticherling

Department of Cardiology/Electrophysiology, University Hospital

Basel, 4031 Basel, Switzerland

e-mail: nikolap12@yahoo.com
Conclusion Adding a simple and fast anatomical reconstruction of the transseptal access to the standard left atrial mapping procedure allows for easy and fluoroscopy-free recrossing of the interatrial septum during atrial fibrillation ablation and further reduces radiation exposure.

Keywords Pulmonary vein isolation · Atrial fibrillation . Electroanatomic mapping system $\cdot$ Fluoroscopy reduction
Abbreviations
AF Atrial fibrillation
BMI Body mass index
EF Electrophysiology fellow
EO Experienced operator
IQR Interquartile range
LA Left atrium
PVI Pulmonary vein isolation
PFO Patent foramen ovale
TSP Transseptal puncture

\section{Introduction}

In the last decade, pulmonary vein isolation (PVI) emerged as a therapy for many patients with atrial fibrillation (AF). Since then, the number of PVIs increased substantially not least due to development of electroanatomic mapping (EAM) systems [1]. However, fluoroscopy is still used for performing transseptal punctures (TSP), catheter placement, and handling of the sheaths [2]. Use of the fluoroscopy exposes the operators, the nurses, and the patients to ionizing radiation. Efforts have been made to reduce radiation exposure to the patients and the electrophysiology staff. Today, using contemporary EAM systems, pulmonary vein isolation can be performed with low doses of fluoroscopy. Standard, fluoroscopy-guided 
transseptal puncture is performed in the majority of cases [3]. However, echocardiographic (intracardiac or transesophageal echocardiography) are being used to increase patient safety and reduce fluoroscopy exposure $[4,5]$. Also, in the PVI era, advances in electroanatomical mapping systems have dramatically reduced fluoroscopy times during the TSP and PVI. In experienced hands, fluoroscopy today is needed almost only for the initial transseptal puncture. However, inadvertent loss of left atrial access during the ablation procedures can occur and regaining access can be time-consuming and increase the radiation exposure to the patient and the operator. Furthermore, during persistent $\mathrm{AF}$ ablation, mapping and ablation in the right atrium might be needed $[6,7]$. In some cases, after right atrial ablation, new left atrial access is needed to complete the ablation.

We sought to evaluate the feasibility and safety of recrossing the interatrial septum using a simple electroanatomical landmark without the use of fluoroscopy.

\section{Methods}

\subsection{Study population}

The study population consisted of 25 consecutive patients undergoing PVI. Indications for PVI were according to current ESC and EHRA guidelines for atrial fibrillation [8]. We included patients undergoing PVI for paroxysmal and persistent AF. Written informed consent was obtained for PVI in all patients.

\subsection{Preprocedural management}

All patients provided informed consent before the procedure. Atrial fibrillation was verified in a 12-lead ECG in all patients. All subjects underwent transesophageal echocardiography to rule out left atrial thrombus the day before the procedure. Left atrial anatomy was assessed in all patients using cardiac magnetic resonance imaging or computed tomography prior to the procedure. Oral anticoagulation was not interrupted for the procedure in patients on vitamin $\mathrm{K}$ antagonists. In patients on dabigatran, the last dose was given $24 \mathrm{~h}$ before the procedure.

\subsection{Transseptal puncture}

After obtaining vascular access in the right groin, one short (8 F) and two long sheaths (SL1, 8.5 F, St. Jude Medical, MN, USA) were placed over the long, $\mathrm{J}$ tip wires placed in the superior vena cava. After vascular access, $2500 \mathrm{IU}$ of heparin were administered. The ablation catheter (Thermocool SF or SmartTouch, Biosense Webster, USA) was then positioned through the short sheath in the coronary sinus in order to serve as a landmark for TSP. TSP was performed according to our standard protocol. Using biplane fluoroscopy, two transseptal punctures were made with the use of contrast injection and pressure monitoring from the tip of the needle. Once the puncture was made, the $0.032 \mathrm{~F} \mathrm{~J}$ tip wire was advanced into one of the left pulmonary veins and the whole system was advanced over the wire. For the purpose of this study, each transseptal puncture was then categorized according to difficulty as easy, medium, or difficult. If TSP was successful with the first attempt, with no need for repositioning of the sheaths or a repeated pulldown and it was easy to get across with the sheath, it was categorized as easy. If there was a need for a repeated pulldown and repositioning or there were difficulties with advancing the system across the septum, the puncture was categorized as medium difficult. If both repositioning or repeated pulldown maneuvers were needed together with difficulties in advancing the system across the septum, the puncture was termed difficult. After the successful TSP, heparin was administered to achieve an activated clotting time $(\mathrm{ACT})>350 \mathrm{~s}$. The ACT was checked every 20 min during the whole procedure.

\subsection{Electroanatomical mapping}

After left atrial access, fast anatomical mapping (FAM) of the left atrium (LA) was performed using the EAM system. The LA reconstruction from the cardiac MRI was used as guidance. FAM was performed using a 20 electrode variable circular mapping catheter (Lasso Nav, Biosense Webster, Diamond Bar, CA, USA) with additional FAM reconstruction with the ablation catheter if needed. After completion of the LA map, additional anatomical mapping of the transseptal access site was created. While assuring that the transseptal sheath was in the LA, the ablation catheter was retracted to the level of the inferior vena cava to mark the direction of the TSP and the sheath ((Fig. 1a) showing right anterior oblique (RAO) and left anterior oblique (LAO) views with superior tilt on the CARTO map). The procedure of mapping the TSP site added less than $1 \mathrm{~min}$ to the standard PVI procedure in all cases.

\subsection{Pulmonary vein isolation}

PVI was then performed with the standard protocol using the 3.5-mm irrigated-tip ablation catheter (ThermoCool SF, SmartTouch, Biosense Webster, Diamond Bar, CA, USA) with power set at 25 to $30 \mathrm{~W}$ and flow rate of $17 \mathrm{~mL} / \mathrm{min}$. Ablation was performed with continuous, encircling lesions in an antral manner. Electrical isolation was confirmed with the 20 pole circular mapping catheter by verifying entrance and/or exit block in all PVs. 


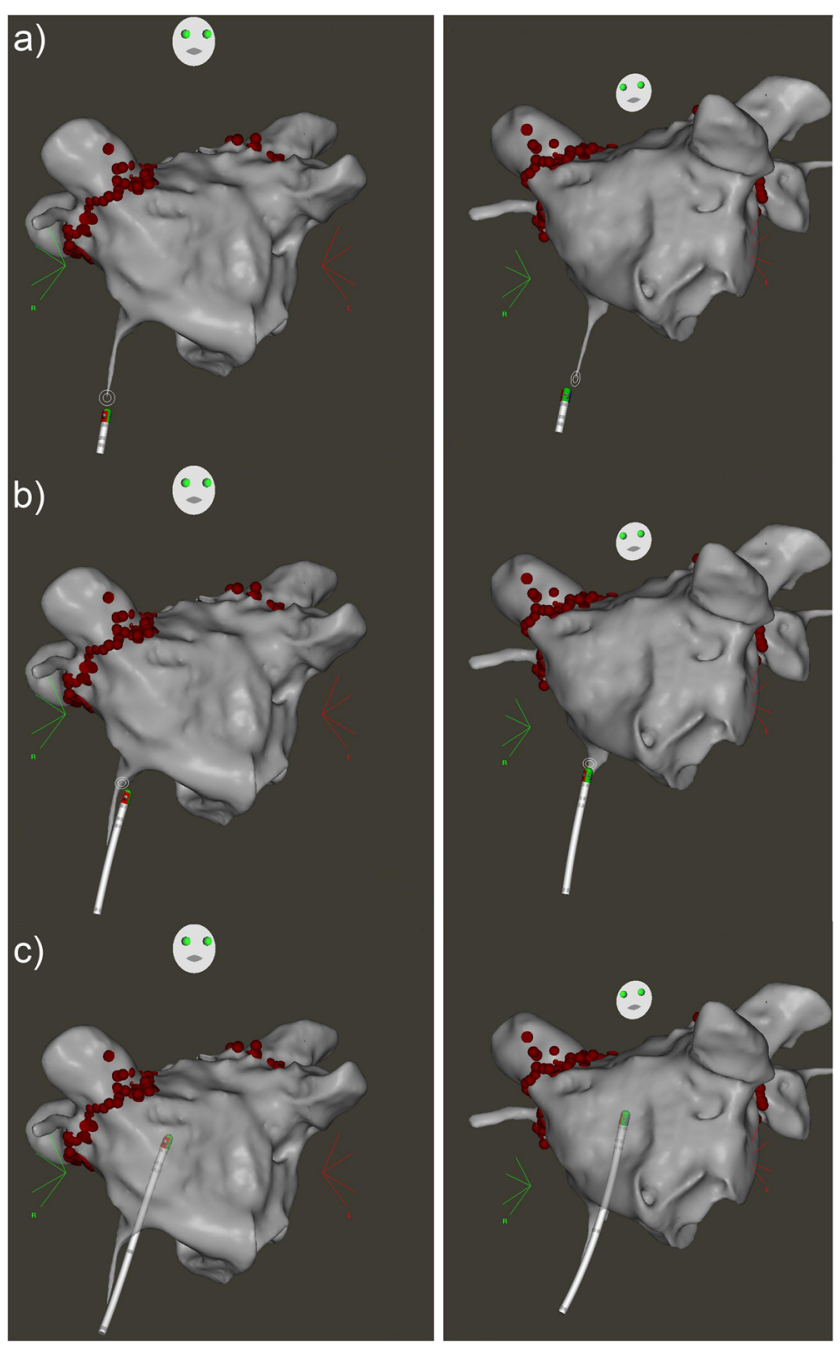

Fig. 1 RAO view is shown on the left and LAO view with superior tilt on the right. a-c Recrossing of the interatrial septum with catheter positions in inferior vena cava (a), at the interatrial septum (b), and in the left atrium after recrossing (c)

\subsection{Recrossing the interatrial septum}

Once the veins were isolated, transseptal sheaths and catheters were pulled back from the LA to the inferior vena cava. At that time, recrossing the interatrial septum with the ablation catheter was attempted by the senior, experienced operator (EO) and by the electrophysiology fellow (EF). Recrossing was done without the use of fluoroscopy, only with the guidance of the previously recorded "transseptal sleeve" on the FAM. Standard right anterior oblique (RAO) $30^{\circ}$ and left anterior oblique (LAO) $45^{\circ}$ projections were used on EAM (Fig. 1). Both, successful recrossing and time to recross were recorded. During some of the PVI procedures, recrossing was also attempted using the same method due to catheter dislodgement to the right atrium or mapping in the right atrium. In these cases, recrossing was performed using the above-described method, but only success rate was recorded, without timing.

\subsection{Postprocedural management}

After sheath removal, all patients were monitored overnight. Transthoracic echocardiography was performed after the procedure to rule out pericardial effusion. Oral anticoagulation was continued after the procedure for at least 3 months and after that according to CHA2DS2VASC score criteria. Patients were discharged at the first postoperative day if no complications occurred.

\subsection{Statistical analysis}

Continuous variables are presented as mean \pm one standard deviation or as median and interquartile range (IQR) in case of skewed distribution. For continuous variables, comparisons were made using Student's $t$-test, or Mann-Whitney $U$ test, as appropriate. A one-way ANOVA with Tukey's post hoc test was used for comparisons between multiple groups. A $p$ value $<0.05$ was considered to indicate statistical significance. Calculations were made using GraphPad Prism (5.0a).

\section{Results}

\subsection{Study population}

A total of 25 patients (mean age of $58 \pm 7$ years, $76 \%$ men, left atrial size in the parasternal long axis $41 \pm 6 \mathrm{~mm}$, mean left ventricular ejection fraction $54 \pm 6 \%$ ) were studied.

Twelve patients (48 \%) underwent PVI for paroxysmal atrial fibrillation and $13(52 \%)$ for persistent AF. Median duration of AF was 24 months (IQR 14.8-50.3). Baseline clinical characteristics of patients are shown in Table 1.

\subsection{Procedural data}

In all patients PVI was performed using the $3.5-\mathrm{mm}$ irrigated tip catheter (Thermocool SF, Biosense Webster, Diamond Bar, CA, USA). Acute pulmonary vein isolation was achieved in all patients. Median procedure duration and mean ablation

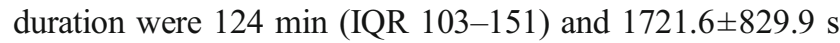
respectively. Mean fluoroscopy time was $8.9 \pm 6.7 \mathrm{~min}$ and median fluoroscopy doses (dose area product) was $1245 \mu_{\mathrm{Gym}^{2}}$ (IQR 659-1939), respectively. No periprocedural complications occurred.

\subsection{Recrossing of the interatrial septum}

Recrossing of the interatrial septum was successfully performed both by electrophysiology fellows and experienced operators in all procedures in median $14 \mathrm{~s}$ (IQR 7-20). Median recrossing times did not differ significantly between 
Table 1 Shows baseline characteristics of the patients

\begin{tabular}{ll}
\hline Number of patients & 25 \\
Age (years) & $58 \pm 7$ \\
BMI $\left(\mathrm{kg} / \mathrm{m}^{2}\right)$ & $28 \pm 4$ \\
Male $(\%)$ & $19(76)$ \\
Female $(\%)$ & $6(24)$ \\
Types of AF & \\
Paroxysmal (\%) & $12(48)$ \\
Persistent (\%) & $13(52)$ \\
History of AF (months) & $24(14.8-50.3)$ \\
Prior antiarrhythmic drugs & $1.2 \pm 0.7$ \\
Hypertension & $20(80)$ \\
Diabetes mellitus & $2(8)$ \\
Coronary artery disease & $4(16)$ \\
Echocardiography & \\
Left atrial diameter (PLAX) & $41 \pm 6$ \\
Left atrial volume (mL) & $36.8 \pm 11.2$ \\
LVEF & $54 \pm 6$ \\
PFO & $3(12)$ \\
CHA2DS2vasc score & $1.5 \pm 1$ \\
HAS BLED score & $1(0-1)$ \\
EHRA score I/II/III/IV & $2 / 18 / 5 / 0$ \\
\end{tabular}

Numbers are given as mean \pm standard deviation, as median and interquartile range or as number and percentage

easy TSP group and medium/difficult TSP group (Table 2). Since there was a small number of TSPs that were classified as medium or difficult, they were analyzed as a single group. Median times to recross the septum did not differ significantly between EF and EO (14 s (IQR 8.0-26.5) versus $12 \mathrm{~s}$ (IQR $6.5-17.5), p=0.26$ ). Procedural data and results of recrossing the interatrial septum using this method are shown in Table 2. In five patients $(20 \%)$, catheter displacement into the right atrium occurred during the PVI procedure and in all five cases recrossing was successfully performed using the EAM of the TSP without fluoroscopy.

\section{Discussion}

This study shows that recrossing the interatrial septum using an anatomical map of the transseptal puncture is safe, feasible, and can be learned easily. Recrossing using this method was achieved in a median of $14 \mathrm{~s}$ (IQR 7-20) and was successfully performed both by experienced operators and fellows. Marking the FAM of the TSP after the creation of the FAM of the LA adds less than $1 \mathrm{~min}$ to the procedure, and it can later serve as a marker for recrossing the interatrial septum in case of catheter dislodgement to the right atrium or after right atrial mapping. This not only potentially reduces procedure times but more
Table 2 Procedural data in our study patients

\begin{tabular}{ll}
\hline Procedural data & \\
Number of procedures & 25 \\
PVI number & $1.6 \pm 0.6$ \\
Procedure duration (min) & $124(103-151)$ \\
Fluoroscopy time (min) & $8.9 \pm 6.7$ \\
Dose area product $\left(\mu \mathrm{Gym}^{2}\right)$ & $1245(659-1939)$ \\
Total ablation time (s) & $1721.6 \pm 829.9$ \\
Complications & 0 \\
Recrossing data & \\
Number of transseptals & 2 \\
Transseptal difficulty $1 / 2 / 3$ & $19 / 3 / 3$ \\
Recrossing success total & $50 / 50$ \\
Transseptal success EF & $25 / 25$ \\
Transseptal success EO & $25 / 25$ \\
Time for recrossing total (s) & $14(7-20)$ \\
Time for recrossing EF & $14(8-26.5)^{*}$ \\
Time for recrossing EO & $12(6.5-17.5)^{*}$ \\
Time for recrossing depending on difficulty & \\
of TSP total (s) & $13(7.0-18.5)^{* *}$ \\
Easy & $18(13.8-49.5)^{* *}$ \\
Medium and difficult & 0 \\
Complications &
\end{tabular}

Numbers are given as mean \pm standard deviation or as median and interquartile range

${ }^{*} p=0.26$ for recrossing times between experienced operator and fellow; $* * p=0.328$ between easy TSP and medium and difficult combined

importantly reduces fluoroscopy times during PVI. To our knowledge, this is the first prospective evaluation of feasibility of fluoroscopy-free manual recrossing of the interatrial septum with the use of electroanatomic mapping system.

Although fluoroscopy still remains indispensable for most of the electrophysiology procedures, especially when TSP is involved, efforts have been made to reduce radiation exposure to patients and operators. Radiation exposure has potential detrimental health effects on both operators and patients both through stochastic effects (including malignancy) and deterministic effects (skin erythema, cataracts) [9]. Applying the ALARA concept ("As low as reasonably achievable") in a daily practice is recommended by most cardiology and radiology societies $[9,10]$.

The technique we used can be considered as a modification and advance of that described by Yamada et al. [11] with a one-puncture, double-transseptal catheterization maneuver. In their report, they performed a single TSP, and while leaving a guide wire across the interatrial septum in the left-sided PVs, crossed the septum with the mapping catheter beside the guide wire. While the approach described by Yamada et al. used fluoroscopy and a wire left in the LA as a landmark, our approach is completely fluoroscopy-free and uses an anatomical map to guide access to the LA. 
During the PVI procedures, access to the LA can be lost due to catheter/sheath slipping in the right atrium, especially in inexperienced hands. On the other, hand complex ablations for persistent $\mathrm{AF}$ include mapping of both left and right atria for CFAEs and lines. In these cases, regaining LA access can be time-consuming and increases procedure and fluoroscopy duration. Reaccess to the left atrium can be achieved either by attempting to advance the ablation catheter through the septum guided by fluoroscopy or to repeat the transseptal puncture. Both of these techniques require fluoroscopy. Since PVI is the procedure with the highest radiation doses in electrophysiology [10] efforts should be made to reduce radiation exposure. Reduction has already been achieved with the use of electroanatomic mapping systems [12, 13], but additional reduction should be a goal in every procedure.

Recently, Nguyen et al. [14] described recrossing of the interatrial septum in five patients using a remote magnetic navigation system and electroanatomic mapping system. Transseptal recrossing was successfully performed in all patients with no complications.

We showed that the interatrial septum can be readily recrossed without the use of fluoroscopy in all of our 25 patients. While creating the FAM of the TSP and the sheath added less than a minute to the procedure, recrossing was successfully accomplished in a median of $14 \mathrm{~s}$ and could be easily learned by the fellows. The procedure could be performed safely and no complications occurred due to the recrossing procedure. This is an important aspect because in contrast to the soft and floppy ablation catheters used during PVI with magnetic navigation (e.g., Thermocool RMT), the ablation catheters used in this study (Thermocool SF and Smart Touch) are relatively stiff at the tip. Contact force information may even be used to prevent the operator from using extensive force when attempting to recross the interatrial septum. However, this was not analyzed systematically in this study.

\section{Study limitations}

A relatively small number of patients were included in our study. Even though we have not experienced complications using this method, a recent study done by Lehrmann et al. [15] showed incidence of $1.5 \%$ of interatrial septum dissection (IASD) and/or perforation when a catheter probing or trans patent foramen ovale (PFO) technique was used for transseptal access. However, in our patient series, it was feasible and safe to recross the interatrial septum in all patients twice, by senior operators and fellows without any significant difference in the time required to recross. Also, we included patients with a first PVI procedure as well as repeat procedures and also patients in whom there were difficulties with transseptal puncture. Therefore, we can assume that this procedure can be repeated in most patients undergoing PVI. On the other hand, we performed all procedures with one of the available electroanatomic mapping system and all ablations (and recrossings) with the same catheter type. Further evaluations are needed with different mapping systems and ablation catheters. Although recrossing with a different mapping system has been described [14], it was performed with remote a magnetic navigation system, and in a smaller number of patients.

\section{Conclusion}

Adding a simple and fast electroanatomical reconstruction of the transseptal access to the standard left atrial mapping procedure allows for easy and fluoroscopy-free recrossing of the interatrial septum during atrial fibrillation ablation and further reduces radiation exposure during PVI.

\section{References}

1. Cappato, R., Calkins, H., Chen, S.-A., Davies, W., Iesaka, Y., Kalman, J., et al. (2010). Updated worldwide survey on the methods, efficacy, and safety of catheter ablation for human atrial fibrillation. Circulation. Arrhythmia and Electrophysiology, 3(1), 32-38.

2. Calkins, H., Kuck, K. H., Cappato, R., Brugada, J., Camm, A. J., Chen, S.-A., et al. (2012). 2012 HRS/EHRA/ECAS expert consensus statement on catheter and surgical ablation of atrial fibrillation: recommendations for patient selection, procedural techniques, patient management and follow-up, definitions, endpoints, and research trial design: a report of the Heart Rhythm Society (HRS) Task Force on Catheter and Surgical Ablation of Atrial Fibrillation. Developed in partnership with the European Heart Rhythm Association (EHRA), a registered branch of the European Society of Cardiology (ESC) and the European Cardiac Arrhythmia Society (ECAS); and in collaboration with the American College of Cardiology (ACC), American Heart Association (AHA), the Asia Pacific Heart Rhythm Society (APHRS), and the Society of Thoracic Surgeons (STS). Endorsed by the governing bodies of the American College of Cardiology Foundation, the American Heart Association, the European Cardiac Arrhythmia Society, the European Heart Rhythm Association, the Society of Thoracic Surgeons, the Asia Pacific Heart Rhythm Society, and the Heart Rhythm Society. Journal of Interventional Cardiac Electrophysiology, 33, 171-257.

3. De Ponti, R., Cappato, R., Curnis, A., Della Bella, P., Padeletti, L., Raviele, A., et al. (2006). Transseptal catheterization in the electrophysiology laboratory: data from a multicenter survey spanning 12 years. Journal of the American College of Cardiology, 47(5), $1037-1042$.

4. Tucker, K. J., Curtis, A. B., Murphy, J., Conti, J. B., Kazakis, D. J., Geiser, E. A., et al. (1996). Transesophageal echocardiographic guidance of transseptal left heart catheterization during radiofrequency ablation of left-sided accessory pathways in humans. Pacing and Clinical Electrophysiology: PACE, 19(3), 272-281.

5. Daoud, E. G., Kalbfleisch, S. J., \& Hummel, J. D. (1999). Intracardiac echocardiography to guide transseptal left heart 
catheterization for radiofrequency catheter ablation. Journal of Cardiovascular Electrophysiology, 10(3), 358-363.

6. Hunter, R. J., Diab, I., Tayebjee, M., Richmond, L., Sporton, S., Earley, M. J., et al. (2011). Characterization of fractionated atrial electrograms critical for maintenance of atrial fibrillation a randomized, controlled trial of ablation strategies (the CFAE AF trial). Circulation. Arrhythmia and Electrophysiology, 4(5), 622-629.

7. O’Neill, M. D., Wright, M., Knecht, S., Jaïs, P., Hocini, M., Takahashi, Y., et al. (2009). Long-term follow-up of persistent atrial fibrillation ablation using termination as a procedural endpoint. European Heart Journal, 30(9), 1105-1112.

8. Camm, A. J., Lip, G. Y. H., De Caterina, R., Savelieva, I., Atar, D., Hohnloser, S. H., et al. (2012). 2012 focused update of the ESC Guidelines for the management of atrial fibrillation: an update of the 2010 ESC Guidelines for the management of atrial fibrillation. Developed with the special contribution of the European Heart Rhythm Association. European Heart Journal, 33(21), 2719-2747.

9. Natarajan, M. K., Paul, N., Mercuri, M., Waller, E. J., Leipsic, J., Traboulsi, M., et al. (2013). Canadian Cardiovascular Society position statement on radiation exposure from cardiac imaging and interventional procedures. Canadian Journal of Cardiology, 29(11), 1361-1368.

10. Picano, E., Vañó, E., Rehani, M. M., Cuocolo, A., Mont, L., Bodi, V., et al. (2014). The appropriate and justified use of medical radiation in cardiovascular imaging: a position document of the ESC Associations of Cardiovascular Imaging, Percutaneous Cardiovascular Interventions and Electrophysiology. European Heart Journal, eht394.

11. Yamada, T., McElderry, H. T., Epstein, A. E., Plumb, V. J., \& Kay, G. N. (2007). One-puncture, double-transseptal catheterization manoeuvre in the catheter ablation of atrial fibrillation. Europace, 9(7), 487489.

12. Sporton, S. C., Earley, M. J., Nathan, A. W., \& Schilling, R. J. (2004). Electroanatomic versus fluoroscopic mapping for catheter ablation procedures: a prospective randomized study. Journal of Cardiovascular Electrophysiology, 15(3), 310-315.

13. Rotter, M., Takahashi, Y., Sanders, P., Haïssaguerre, M., Jaïs, P., Hsu, L.-F., et al. (2005). Reduction of fluoroscopy exposure and procedure duration during ablation of atrial fibrillation using a novel anatomical navigation system. European Heart Journal, 26(14), 1415-1421.

14. Nguyen, B., Merino, J., Shachar, Y., Estrada, A., Doiny, D., Castrejon, S., et al. (2013). Non-fluoroscopic transseptal catheterization during electrophysiology procedures using a remote magnetic navigation system. JAFIB: Journal of Atrial Fibrillation, 6(4), 6-9.

15. Lehrmann, H., Schneider, J., Jadidi, A. S., Park, C.-I., SchiebelingRömer, J., Allgeier, J., et al. (2014). Transseptal access for left atrial ablation: the catheter-probing techniques are not without risk. Journal of Cardiovascular Electrophysiology, 25, 479-484. 